\title{
Large elastoplastic deformation theory for soils
}

\author{
Koichi HASHIGUCI \\ Dr. Eng and Dr. Agr., Professor, Dept. Prod. Environment. Sci., Kyushu University, \\ Hakozaki, Higashi-ku, Fukuoka 812-8581
}

\begin{abstract}
Constitutive equation for the large elastoplastic deformation of soils is formulated based on the corotational logarithmic (Hencky) strain rate tensor proposed by Naghdabadi and Saidi ${ }^{1}$ and its extension to the tangential-subloading surface model ${ }^{2)-6)}$ proposed by Hashiguchi et al. ${ }^{7)}$. Here, the hyper-elastic constitutive equation of soils is formulated by incorporating the $\ln v-\ln p$ linear relation ${ }^{8)-11)}$ for the isotropic consolidation and the shear modulus depending on the pressure modifying the Junbu's equation ${ }^{12)}$.

Key Words: elastoplasticity, large deformation, logarithmic strain, subloading surface model
\end{abstract}

\section{Introduction}

A Constitutive equation for the elastoplastic deformation has to be formulated so as to fulfill the following requirements.

1 ) The principle of material-frame indifference ${ }^{13)}$ is fulfilled. It is attained by describing constitutive equations in terms of tensors possessing the $o b$ jectivity.

2 ) A strain rate measure is derived by the time-differentiation of an appropriate strain measure which is a function of the deformation gradient describing the relation between the reference and the current configurations of material particles. On the other hand, a stress measure has to be work conjugate with a strain rate measure. Therefore, a stress measure would have to be the one defined on the base of reference configuration.

3 ) An elastic part of strain rate is formulated as the hyper-elasticity in which an elastic strain is induced from a strain energy function.

The stretching tensor, i.e. the symmetric part of the velocity gradient is not appropriate for the strain rate measure since it cannot be derived from any strain measure and is difficult to bring about the hyperelasticty. On the other hand, the Lee's multicative decomposition $^{14)}$ satisfies all the above-mentioned requirements but falls within the framework of the conventional plasticity premising that the interior of yield surface is a purely elastic domain and thus it cannot describe the plastic deformation due to the rate of stress inside the yield surface, needless to say, incapable of describing a cyclic loading behavior.

The large deformation theory proposed by Naghdabadi and Saidi ${ }^{1)}$ adopts the corotational logarithmic (Hencky) strain rate of the left stretch tensor. It has the capability to fulfill the afore-mentioned three requirements. On the other hand, the subloading surface model ${ }^{2), 3)}$ is capable of describing the plastic strain rate due to the change of stress inside the yield surface falling within the unconventional plasticity ${ }^{15}$ and has been extended so as to describe the inelastic strain rate due to the stress rate component tangential to the subloading surface, while it is called the tangential stress rate effect ${ }^{4)-6}$ ). The extended constitutive equation was formulated by Hashiguchi et al. ${ }^{7}$ generalizing the large deformation theory of Naghdabadi and Saidi ${ }^{1)}$ for metals to the theory for general materials and incorporating it into the tangentialsubloading surface model of Hashiguchi ${ }^{2)-6}$.

In this article, the large deformation theory of Hashiguchi et al. ${ }^{7)}$ is first refined such that the Cauchy stress is replaced to the Kircchhoff stress which is the work conjugate with the Hencky strain 
rate and the elastic rotation is not ignored. Based on it the constitutive equation for the large elastoplastic deformation of soils is formulated. Here, the $h y$ per-elastic constitutive equation of soils is formulated by incorporating the isotropic consolidation of $\ln v$ - $\ln p$ linear relation ${ }^{8)-11)}$ and the shear modulus depending on the elastic volumetric strain modifying the Junbu's equation ${ }^{12)}$.

The signs of a stress (rate) and a strain rate (a symmetric part of velocity gradient) components are chosen to be positive for tension, and the stress for soils is meant to be the effective stress, i.e. the stress excluded a pore pressure from the total stress throughout this article.

\section{Constitutive Equation}

The deformation gradient $\mathbf{F}$ can be led to the polar decomposition:

$$
\mathbf{F} \equiv \frac{\partial \mathbf{x}}{\partial \mathbf{X}}=\mathbf{V R}
$$

where $\mathbf{x}$ and $\mathbf{X}$ are the position vectors of material particle in the current and the reference configuration, respectively, and

$$
\mathbf{V}^{2}=\mathbf{F F} \mathbf{F}^{\mathrm{T}}, \mathbf{R}=\mathbf{V}^{-1} \mathbf{F},
$$

()$^{-1}$ stands for the inverse tensor, whilst the left stretch tensor $\mathbf{V}$ can be written in the principal directions as

$$
\mathbf{V}=\sum_{\alpha=1}^{3} \lambda_{\alpha} \mathbf{n}_{\alpha} \otimes \mathbf{n}_{\alpha},
$$

denoting the principal values and directions as $\lambda_{\alpha}$ and $\mathbf{n}_{\alpha}$, respectively.

Throughout this paper the corotational rate $\stackrel{\circ}{\mathbf{T}}$ with objectivity for an arbitrary second-order tensor $\mathbf{T}$ is given as

$$
\stackrel{\circ}{\mathbf{T}} \equiv \stackrel{\circ}{\mathbf{T}}-\boldsymbol{\Omega}^{m} \mathbf{T}+\mathbf{T} \boldsymbol{\Omega}^{m},
$$

where $\left({ }^{\bullet}\right)$ stands for the material-time derivative and $\boldsymbol{\Omega}^{m}$ is the proper spin tensor of material-substructure.

Let the logarithmic (Hencky) strain rate $(\ln \mathbf{V})^{\circ}$ be additively decomposed into the elastic strain rate $\left((\ln \mathbf{V})^{\circ}\right)^{e}$ and the inelastic strain rate $\left((\ln \mathbf{V})^{\circ}\right)^{i}$, i.e.

$$
(\ln \mathbf{V})^{\circ}=\left((\ln \mathbf{V})^{\circ}\right)^{e}+\left((\ln \mathbf{V})^{\circ}\right)^{i}
$$

Further, let the inelastic strain rate $\left((\ln \mathbf{V})^{\circ}\right)^{i}$ be additively decomposed into the plastic strain rate $\left((\ln \mathbf{V})^{\circ}\right)^{p}$ and the tangential strain rate $\left((\ln \mathbf{V})^{\circ}\right)^{t}$, i.e.

$$
\left((\ln \mathbf{V})^{\circ}\right)^{i}=\left((\ln \mathbf{V})^{\circ}\right)^{p}+\left((\ln \mathbf{V})^{\circ}\right) t
$$

provided that $\left((\ln \mathbf{V})^{\circ}\right)^{p}$ and $\left((\ln \mathbf{V})^{\circ}\right)^{t}$ are induced by the stress rate component normal and tangential, respectively, to the yield and/or loading surface.

Assume that the elastic logarithmic strain $(\ln \mathbf{V})^{e}$ is derived from the following strain energy function $\Psi\left((\ln \mathrm{V})^{e}\right)$, i.e.

$$
\boldsymbol{\tau}=\frac{\partial \Psi\left((\ln \mathbf{V})^{e}\right)}{\partial(\ln \mathbf{V})^{e}},
$$

where $\boldsymbol{\tau}$ is the Kirchhoff stress. The material-time derivative of Eq. (7) leads to

$$
\dot{\boldsymbol{\tau}}=\mathbf{E}\left((\ln \mathbf{V})^{e}\right)^{\bullet}
$$

putting the elastic modulus $\mathbf{E}$ as

$$
\mathbf{E}=\frac{\partial^{2} \Psi\left((\ln \mathbf{V})^{e}\right)}{\partial\left\{(\ln \mathbf{V})^{e}\right\}^{2}} .
$$

Eq. (8) can be transformed to the corotational derivative as follows (see Appendix):

$$
\stackrel{\circ}{\mathbf{\tau}}=\mathbf{E}\left((\ln \mathbf{V})^{e}\right)^{\circ}
$$

The following tensors may be substituted for the spin tensor $\boldsymbol{\Omega}^{m}$ of material-substructure.

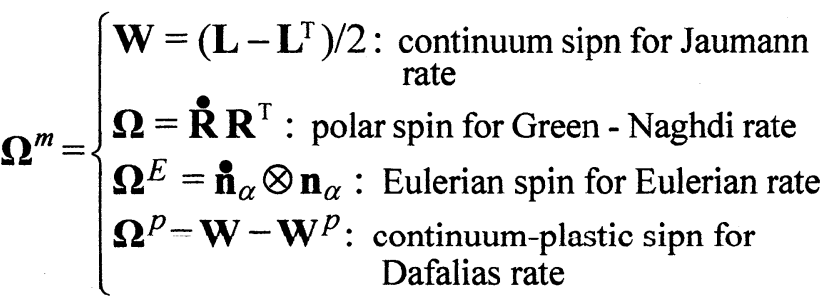

where ()$^{\mathrm{T}}$ denotes the transpose, and

$$
\begin{gathered}
\mathbf{L} \equiv \dot{\mathbf{F}} \mathbf{F}^{-1}, \\
\mathbf{W}^{p} \equiv \varsigma\left\{\boldsymbol{\tau}\left((\ln \mathbf{V})^{\circ}\right)^{p}-\left((\ln \mathbf{V})^{\circ}\right)^{p} \boldsymbol{\tau}\right\},
\end{gathered}
$$

$\checkmark$ is the material parameter. The spin tensor $\boldsymbol{\Omega}^{p}$ was proposed by Dafalias ${ }^{16}$ ) and Eq. (13) for the plastic spin tensor $\mathbf{W}^{p}$ was proposed by Zbib and Aifantis $^{17)}$.

Let the following yield condition be assumed.

$$
f(\boldsymbol{\tau}, \mathbf{H})=F(H),
$$

where the scalar $H$ and the second-order tensor $\mathbf{H}$ are the isotropic and the anisotropic hardening variables, respectively. For soils $H$ is given by the plastic 


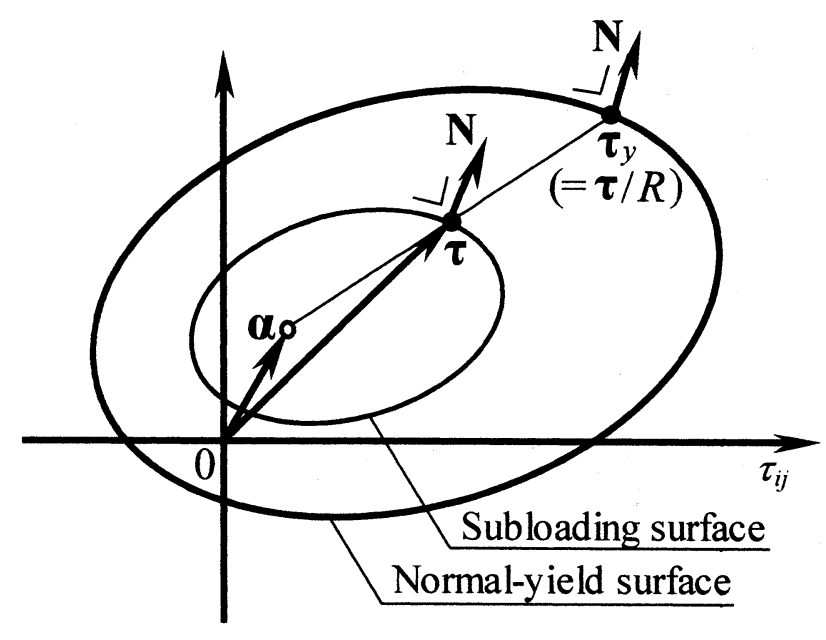

Fig. 1 Normal-yield and subloading surfaces.

volumetric strain and $\mathbf{H}$ is given by the rotational hardening variable ${ }^{18), 19)}$. The function $f$ is assumed to be homogeneous of degree one in the stress $\boldsymbol{\tau}$. Then, if $\mathbf{H}=$ const., the yield surface keeps a similar shape.

Drucker ${ }^{15)}$ defined the unconventional plasticity as the extended plasticity such that the interior of the yield surface is not a purely elastic domain but a plastic deformation is induced by the rate of stress inside the yield surface. The most appropriate model falling within the framework of the unconventional plasticity would be the subloading surface model ${ }^{2), 3)}$.

In the subloading surface model the conventional yield surface is renamed as the normal-yield surface, since its interior is not regarded as a purely elastic domain. Then, let the subloading surface be introduced, which always passes through the current stress point $\boldsymbol{\tau}$ and also keeps the shape similar to the normal-yield surface and the orientation of similarity. The similarity and the orientation of similarity of surfaces possess the following geometrical properties.

i) All lines connecting an arbitrary point on or within the subloading surface and its conjugate point on or within the normal-yield surface join at the specific point called the similarity-center.

ii ) All ratios of the length of an arbitrary line-element connecting two points on or inside the subloading surface and that of an arbitrary conjugate line-element connecting two conjugate points on or inside the normal-yield surface are identical. The ratio is called the similarity-ratio which coincides with the ratio of the sizes of these surfaces.

Let the similarity-ratio of the subloading surface to the normal-yield surface be specifically called the normal-yield ratio, and let it be denoted by $R$, where $R=0$ corresponds to the null stress state, $0<R<1$ to the subyield state and $R=1$ to the normal-yield state in which the stress lies on the normal-yield surface. Therefore, the normal-yield ratio $R$ plays the role of the three-dimensional measure for the degree of approach of stress to the normal-yield surface.

Then, the subloading surface is described as

$$
f(\boldsymbol{\tau}, \mathbf{H})=R F(H),
$$

The normal-yield and the subloading surfaces are illustrated in Fig. 1, where $\boldsymbol{\tau}_{y}(\equiv \boldsymbol{\tau} / R)$ is the conjugate stress on the normal-yield surface at which the normalized outward-normal $\mathbf{N}$ is same as that at the current stress $\boldsymbol{\tau}$ on the subloading surface.

The time-differentiation of Eq. (15) leads to

$\operatorname{tr}\left\{\frac{\partial f(\boldsymbol{\tau}, \mathbf{H})}{\partial \boldsymbol{\tau}} \stackrel{\circ}{\boldsymbol{\tau}}\right\}+\operatorname{tr}\left\{\frac{\partial f(\boldsymbol{\tau}, \mathbf{H})}{\partial \mathbf{H}} \stackrel{\circ}{\mathbf{H}}\right\}=\stackrel{R}{R} F+R \stackrel{\circ}{F}$.

Let the following evolution equation of the normal-yield ratio $R$ be assumed.

$\dot{R}=U(R)\left\|\left((\ln \mathbf{V})^{\circ}\right)^{p}\right\|$ for $\left((\ln \mathbf{V})^{\circ}\right)^{p} \neq \mathbf{0}$,

where $U$ is a monotonically decreasing function of the normal-yield ratio $R$ (see Fig. 2), fulfilling the condition

$$
\begin{aligned}
& U=\left\{\begin{array}{l}
\infty \text { for } R=0, \\
0 \text { for } R=1,
\end{array}\right. \\
& (U<0 \text { for } R>1) .
\end{aligned}
$$

Let the function $U$ satisfying Eq. (18) be simply

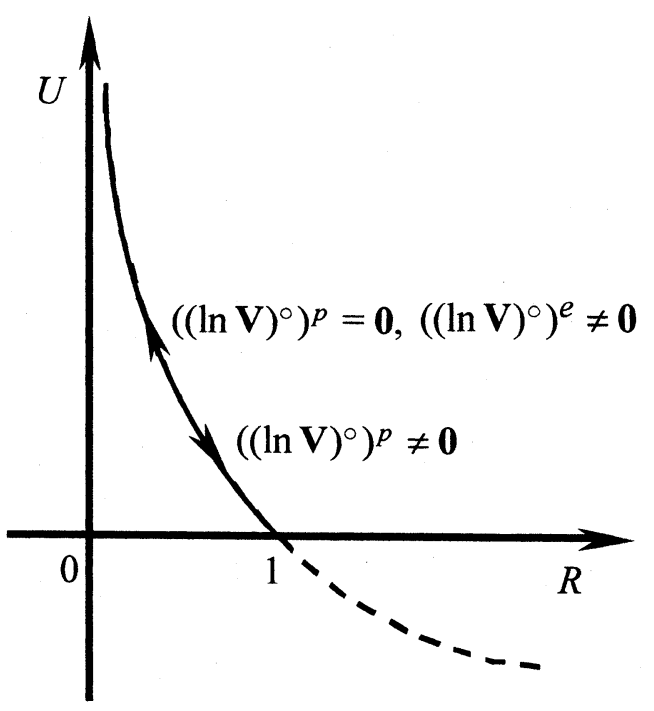

Fig. 2. The function $U$ in the evolution rule of the normal-yield ratio $R$. 
given by

$$
U(R)=-u \ln R
$$

where $u$ is the material constant.

The substitution of Eq. (17) into Eq. (16) leads to the consistency condition extended to the subloading surface:

$$
\begin{aligned}
& \operatorname{tr}\left\{\frac{\partial f(\boldsymbol{\tau}, \mathbf{H})}{\partial \boldsymbol{\tau}} \stackrel{\circ}{\boldsymbol{\tau}}\right\}+\operatorname{tr}\left\{\frac{\partial f(\boldsymbol{\tau}, \mathbf{H})}{\partial \mathbf{H}} \stackrel{\leftrightarrow}{\mathbf{H}}\right\} \\
& =U\left\|\left((\ln \mathbf{V})^{\circ}\right)^{p}\right\| F+R \stackrel{\circ}{F} .
\end{aligned}
$$

Assume the plastic flow rule

$$
\left((\ln \mathbf{V})^{\circ}\right)^{p}=\lambda \mathbf{N},
$$

where $\lambda$ is the positive proportionality factor and

$$
\mathbf{N} \equiv \frac{\partial f(\boldsymbol{\tau}, \mathbf{H})}{\partial \boldsymbol{\tau}} /\left\|\frac{\partial f(\boldsymbol{\tau}, \mathbf{H})}{\partial \boldsymbol{\tau}}\right\| \quad(\|\mathbf{N}\|=1) .
$$

The substitution of Eq. (21) into Eq. (20) leads to

$$
\begin{aligned}
& \operatorname{tr}\left\{\frac{\partial f(\boldsymbol{\tau}, \mathbf{H})}{\partial \boldsymbol{\tau}} \boldsymbol{\tau}\right\}+\operatorname{tr}\left\{\frac{\partial f(\boldsymbol{\tau}, \mathbf{H})}{\partial \mathbf{H}} \lambda \mathbf{h}\right\} \\
& =U \lambda F+R F^{\prime} \lambda h,
\end{aligned}
$$

where

$$
\begin{gathered}
F^{\prime} \equiv d F / d H, \\
h \equiv \stackrel{\circ}{H} / \lambda, \quad \mathbf{h} \equiv \stackrel{\circ}{\mathbf{H}} / \lambda,
\end{gathered}
$$

From Eq. (23) one has

$$
\lambda \equiv \frac{\operatorname{tr}(\mathbf{N} \boldsymbol{\tau})}{M^{p}},
$$

where

$$
M^{p} \equiv\left\{\frac{F^{\prime}}{F} h-\frac{1}{R F} \operatorname{tr}\left(\frac{\partial f(\boldsymbol{\tau}, \mathbf{H})}{\partial \mathbf{H}} \mathbf{h}\right)+\frac{U}{R}\right\} \operatorname{tr}(\mathbf{N} \boldsymbol{\tau})
$$

by use of the following relation based on Euler's theorem for a homogeneous function.

$$
\begin{aligned}
\frac{\partial f(\boldsymbol{\tau}, \mathbf{H})}{\partial \boldsymbol{\tau}}=\left\|\frac{\partial f(\boldsymbol{\tau}, \mathbf{H})}{\partial \boldsymbol{\tau}}\right\| \mathbf{N}=\frac{\operatorname{tr}\left(\frac{\partial f(\boldsymbol{\tau}, \mathbf{H})}{\partial \boldsymbol{\tau}} \boldsymbol{\tau}\right)}{\operatorname{tr}(\mathbf{N} \boldsymbol{\tau})} \mathbf{N} \\
=\frac{f(\boldsymbol{\tau}, \mathbf{H})}{\operatorname{tr}(\mathbf{N} \boldsymbol{\tau})} \mathbf{N}=\frac{R F}{\operatorname{tr}(\mathbf{N} \boldsymbol{\tau})} \mathbf{N} .
\end{aligned}
$$

Eq. (21) with Eq. (26) leads to

$$
\left((\ln \mathbf{V})^{\circ}\right)^{p}=\frac{\operatorname{tr}(\mathbf{N} \tau)}{M^{p}} \mathbf{N}
$$

Further, modifying the tangential strain rate of Hashiguchi $^{4)}$ or Hashiguchi and Tsutsumi ${ }^{5)}$, the tan- gential plastic strain rate was given by Hashiguchi ${ }^{6}$ as

$$
\left((\ln \mathbf{V})^{\circ}\right)^{t}=\frac{1}{T} \mathbf{E}^{-1} \stackrel{\circ}{\boldsymbol{\tau}}_{t}^{*}
$$

where

$$
\begin{gathered}
\stackrel{\circ}{\boldsymbol{\tau}}_{t}^{*}=\stackrel{\circ}{\boldsymbol{\tau}}^{*}-\stackrel{\circ}{\boldsymbol{\tau}}_{n}^{*}, \quad \stackrel{\circ}{\boldsymbol{\tau}}_{n}^{*} \equiv \operatorname{tr}\left(\mathbf{n}^{*} \stackrel{\circ}{\boldsymbol{\tau}}\right) \mathbf{n}^{*}, \\
\boldsymbol{\tau}^{*} \equiv \boldsymbol{\tau}-\tau_{m} \mathbf{I}, \quad \tau_{m} \equiv \frac{1}{3} \operatorname{tr} \boldsymbol{\tau}, \\
\mathbf{n}^{*} \equiv\left(\frac{\partial f(\boldsymbol{\tau})}{\partial \boldsymbol{\tau}}\right)^{*} /\left\|\left(\frac{\partial f(\boldsymbol{\tau})}{\partial \boldsymbol{\tau}}\right)^{*}\right\|=\frac{\mathbf{N}^{*}}{\left\|\mathbf{N}^{*}\right\|}, \\
T=\frac{\xi}{R^{a}}
\end{gathered}
$$

$a$ is a material constant and $\xi$ is a material function of the stress and the plastic internal variables in general. ( )* designates a deviatoric component. $\stackrel{\circ}{\tau}_{t}^{*}$ is called the deviatoric tangential stress rate.

The logarithmic strain rate is given from Eqs. (5), (6), (8), (29) and (30) as follows:

$$
(\ln \mathbf{V})^{\circ}=\mathbf{E}^{-1} \stackrel{\circ}{\boldsymbol{\tau}}+\frac{\operatorname{tr}(\mathbf{N} \stackrel{\circ}{\boldsymbol{\tau}})}{M^{p}} \mathbf{N}+\frac{1}{T} \mathbf{E}^{-1} \stackrel{\circ}{\boldsymbol{\tau}}_{t}^{*} .
$$

from which one has

$$
\begin{gathered}
\operatorname{tr}\left\{\mathbf{N E}(\ln \mathbf{V})^{\circ}\right\}=\operatorname{tr}(\mathbf{N} \stackrel{\circ}{\boldsymbol{\tau}})+\operatorname{tr}(\mathbf{N E N}) \frac{\operatorname{tr}\left(\mathbf{N}{ }^{\circ}\right)}{M^{p}} \\
=\left\{M^{p}+\operatorname{tr}(\mathbf{N E N})\right\} \frac{\operatorname{tr}(\mathbf{N} \stackrel{\circ}{\boldsymbol{\tau}})}{M^{p}}
\end{gathered}
$$

The positive proportionality factor $\lambda$ in the flow rule (21) is expressed in terms of strain rate, rewriting $\lambda$ by $\Lambda$, from Eq. (36) as follows:

$$
\Lambda=\frac{\operatorname{tr}\left\{\mathbf{N E}(\ln \mathbf{V})^{\circ}\right\}}{M^{p}+\operatorname{tr}(\mathbf{N E N})}
$$

The loading criterion is given as follows ${ }^{20), 21 \text { ) }}$

$$
\left.\begin{array}{l}
\left((\ln \mathbf{V})^{\circ}\right)^{p} \neq \mathbf{0}: \Lambda>0 \\
\left((\ln \mathbf{V})^{\circ}\right)^{p}=\mathbf{0}: \Lambda \leq 0
\end{array}\right\}
$$

It holds from Eqs. (35) and (37) that

$$
\stackrel{\circ}{\boldsymbol{\tau}}=\mathbf{E}(\ln \mathbf{V})^{\circ}-\mathbf{E} \frac{\operatorname{tr}\left\{\mathbf{N E}(\ln \mathbf{V})^{\circ}\right\}}{M^{p}+\operatorname{tr}(\mathbf{N E N})} \mathbf{N}-\frac{1}{T} i_{t}^{*}
$$

Eqs. (31) and (39) lead to

$$
\begin{aligned}
\stackrel{\circ}{\boldsymbol{\tau}}_{t}^{*}=\stackrel{\boldsymbol{\tau}}{*}^{*} & -\operatorname{tr}\left[\mathbf { n } * \left\{\mathbf{E}(\ln \mathbf{V})^{\circ}\right.\right. \\
- & \left.\left.\mathbf{E}\left[\frac{\operatorname{tr}\left\{\mathbf{N E}(\ln \mathbf{V})^{\circ}\right\}}{M^{p}+\operatorname{tr}(\mathbf{N E N})}\right] \mathbf{N}\right\}\right] \mathbf{n}^{*} .
\end{aligned}
$$


Substituting Eq. (40) into Eq. (39), one has

$$
\begin{aligned}
\stackrel{\circ}{\boldsymbol{\tau}}= & \mathbf{E}(\ln \mathbf{V})^{\circ}-\mathbf{E} \frac{\operatorname{tr}\left\{\mathbf{N E}(\ln \mathbf{V})^{\circ}\right\}}{M^{p}+\operatorname{tr}(\mathbf{N E N})} \mathbf{N} \\
& -\frac{1}{T} \stackrel{\circ}{\boldsymbol{\tau}}+\frac{1}{T} \dot{\tau}_{m} \mathbf{I}+\frac{1}{T} \operatorname{tr}\left[\mathbf { n } ^ { * } \left\{\mathbf{E}(\ln \mathbf{V})^{\circ}\right.\right. \\
& \left.\left.-\mathbf{E}\left(\frac{\operatorname{tr}\left\{\mathbf{N E}(\ln \mathbf{V})^{\circ}\right\}}{M^{p}+\operatorname{tr}(\mathbf{N E N})}\right) \mathbf{N}\right\}\right] \mathbf{n}^{*}
\end{aligned}
$$

On the other hand, it holds from Eq. (39) that

$\dot{\tau}_{m}=\frac{1}{3}\left\{\operatorname{tr}\left(\mathbf{N}(\ln \mathbf{V})^{\circ}\right)-\frac{\operatorname{tr}\left\{\mathbf{N E}(\ln \mathbf{V})^{\circ}\right\}}{M^{p}+\operatorname{tr}(\mathbf{N E N})} \operatorname{tr}(\mathbf{E N})\right\}$

The substitution of Eq. (42) into Eq. (41) leads to

$$
\begin{aligned}
\stackrel{\imath}{\boldsymbol{\tau}}= & \frac{1}{1+T}\left[T \mathbf{E}(\ln \mathbf{V})^{\circ}+\frac{1}{3} \operatorname{tr}\left\{\mathbf{N}(\ln \mathbf{V})^{\circ}\right\} \mathbf{I}\right. \\
+ & \operatorname{tr}\left\{\mathbf{n}^{*} \mathbf{E}(\ln \mathbf{V})^{\circ}\right\} \mathbf{n}^{*}-\frac{\operatorname{tr}\left\{\mathbf{N E}(\ln \mathbf{V})^{\circ}\right\}}{M^{p}+\operatorname{tr}(\mathbf{N E N})} \\
& \left.\left\{T \mathbf{E N}+\frac{1}{3} \operatorname{tr}(\mathbf{E N}) \mathbf{I}+\operatorname{tr}\left(\mathbf{n}^{*} \mathbf{E N}\right) \mathbf{n}^{*}\right\}\right] .
\end{aligned}
$$

The plastic strain rate (29) is obtained by substituting the associated flow rule (21) into the consistency condition (20) which is obtained by incorporating the evolution rule (17) of the normal-yield ratio $R$ into the time-differentiation (16) of Eq. (15) for the subloading surface with the gradients of internal variables. Then, the plastic loading process develops gradually as the stress approaches the normal-yield surface, exhibiting a smooth elastic-plastic transition. Thus, the subloading surface model fulfills the smoothness condition ${ }^{21)-24)}$ defined as "the stress rate-strain rate relation (or the stiffness tensor) changes continuously for a continuous change of stress state". This can be expressed mathematically as follows:

$$
\begin{aligned}
& \lim _{\delta \boldsymbol{\tau} \rightarrow \mathbf{0}} \frac{\partial \stackrel{\circ}{\boldsymbol{\tau}}\left(\boldsymbol{\sigma}+\partial \boldsymbol{\tau}, \mathbf{S}_{i},(\ln \mathbf{V})^{\circ}\right)}{\partial(\ln \mathbf{V})^{\circ}} \\
& =\frac{\partial \stackrel{\circ}{\boldsymbol{\tau}}\left(\boldsymbol{\tau}, \mathbf{S}_{i},(\ln \mathbf{V})^{\circ}\right)}{\partial(\ln \mathbf{V})^{\circ}},
\end{aligned}
$$

where $\mathbf{S}_{i}(i=1,2,3, \cdots, \mathrm{m})$ denotes collectively scalar- or tensor-valued internal state variables which describe the alteration of the mechanical response due to the irreversible deformation. $\delta()$ stands for an infinitesimal variation and the response of the stress rate to the strain rate in the current state of stress and internal variables is designated by $\stackrel{\circ}{\tau}\left(\tau, \mathbf{s}_{i},(\ln \mathbf{V})^{\circ}\right)$. Therefore, the subloading surface model has the notable advantages as follows:

1 ) A smooth response (e.g. a smooth axial stress-axial logarithmic strain relation in the uniaxial loading) for a smooth monotonic loading is predicted by the subloading surface model. On the other hand, a nonsmooth response is predicted by constitutive models violating the smoothness condition as in the conventional plasticity with the yield surface enclosing a purely elastic domain.

2 ) Only the decision for the sign of the proportionality factor $\Lambda$ in terms of strain rate is required in the loading criterion of the subloading surface model, since the stress always lies on the subloading surface, which now plays the role of the loading surface, whilst the decision as to whether or not the stress lies on the yield surface is not required. On the other hand, the judgment whether or not the yield condition is fulfilled is required in the conventional plasticity.

3 ) A stress is automatically drawn back to the normal-yield surface even if it goes out from that surface since it is formulated that $\dot{R}>0$ for $R<1$ (subyield state) and $\dot{R}<0$ for $R>1$ (over the normal-yield state) in Eq. (17) with the condition (18)

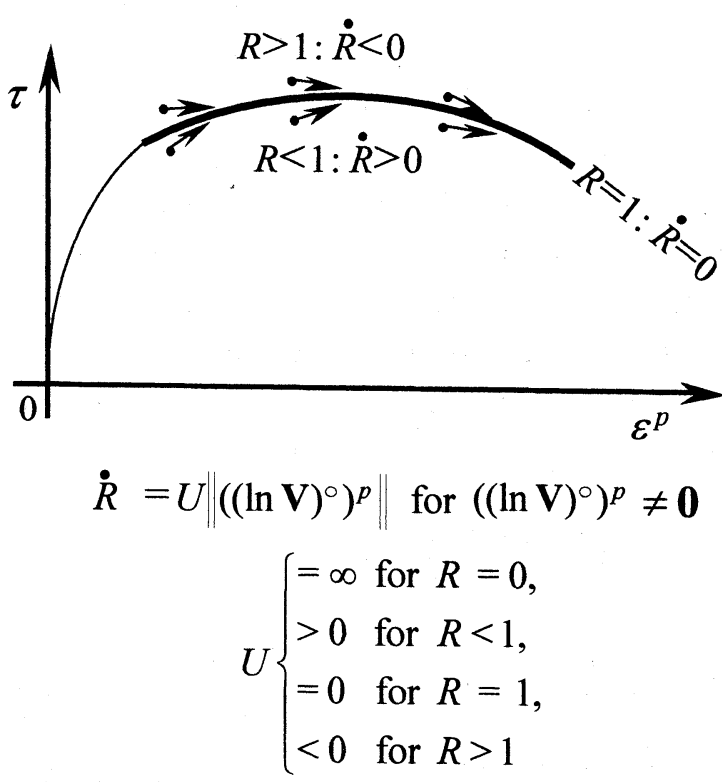

Fig. 3. The stress controlling function contained in the subloading surface model: The stress $\boldsymbol{\tau}$ is automatically controlled so as to approach the normal-yield surface in the plastic-load ing process $\left((\ln \mathbf{V})^{\circ}\right)^{p} \neq \mathbf{0}$. 
(18) (see Fig. 3). Thus, a rough numerical calculation with large loading steps is allowed in the subloading surface model.

Further, the tangential strain rate (30) is also formulated to develop gradually as the' stress approaches the normal-yield surface, fulfilling the continuity condition ${ }^{21-24)}$ defined as "the stress rate response changes continuously for a continuous change of the strain rate" can be expressed mathematically as follows:

$$
\begin{aligned}
\lim _{\delta(\ln \mathbf{V})^{\circ} \rightarrow \mathbf{0}} \stackrel{\circ}{\boldsymbol{\tau}}\left(\boldsymbol{\tau}, \mathbf{S}_{i},\right. & \left.(\ln \mathbf{V})^{\circ}+\delta(\ln \mathbf{V})^{\circ}\right) \\
= & \stackrel{\boldsymbol{\tau}}{ }\left(\boldsymbol{\tau}, \mathbf{S}_{i},(\ln \mathbf{V})^{\circ}\right),
\end{aligned}
$$

whilst the other tangential plasticity models ${ }^{25)-29)}$ violate the continuity condition predicting an abrupt generation of the tangential strain rate when the stress reaches the yield surface.

\section{Constitutive Equation of Soils}

Based on the equations formulated in the previous section, particular forms of material functions for soils are described in this section.

\subsection{Hyper-elastic constitutive equation of soils}

It holds generally that

$$
\begin{aligned}
\operatorname{tr}(\ln \mathbf{V}) & =\sum_{\alpha=1}^{3} \ln \lambda_{\alpha}=\sum_{\alpha=1}^{3} \ln F_{\alpha}=\sum_{\alpha=1}^{3} \ln \frac{\partial x_{\alpha}}{\partial X_{\alpha}} \\
& =\ln \left(\frac{\partial x_{1}}{\partial X_{1}} \frac{\partial x_{2}}{\partial X_{2}} \frac{\partial x_{3}}{\partial X_{3}}\right)=\ln \frac{v}{V},
\end{aligned}
$$

where $V$ and $v$ are the initial and the current volume, respectively. Therefore, the trace of the Hencky strain tensor is equal to the logarithmic volumetric strain. Further, $\operatorname{tr}(\ln \mathbf{V})$ can be additively decomposed into the elastic logarithmic volumetric strain $\operatorname{tr}\left(\ln \mathbf{V}^{e}\right)$ and plastic logarithmic volumetric strain $\operatorname{tr}\left(\ln \mathbf{V}^{p}\right)$ as follows:

$$
\begin{aligned}
& \operatorname{tr}(\ln \mathbf{V})=\ln \left(\frac{\partial x_{1} \partial x_{2} \partial x_{3}}{\partial X_{1} \partial X_{2} \partial X_{3}}\right) \\
& =\ln \left(\frac{\partial x_{1} \partial x_{2} \partial x_{3}}{\partial \bar{x}_{1} \partial \bar{x}_{2} \partial \bar{x}_{3}}\right)+\ln \left(\frac{\partial \bar{x}_{1} \partial \bar{x}_{2} \partial \bar{x}_{3}}{\partial X_{1} \partial X_{2} \partial X_{3}}\right) \\
& =\operatorname{tr}\left(\ln \mathbf{V}^{e}\right)+\operatorname{tr}\left(\ln \mathbf{V}^{p}\right)=\ln \left(\frac{v}{\bar{v}}\right)+\ln \left(\frac{\bar{v}}{V}\right),
\end{aligned}
$$

where $\overline{\mathbf{x}}$ and $\bar{v}$ are the position vector of material particle and the volume in the intermediate configuration unloaded to the initial pressure $p_{0}$. Here, it can be written that

$$
\begin{aligned}
\operatorname{tr}(\ln \mathbf{V}) & =\sum_{\alpha=1}^{3} \ln F_{\alpha}=\sum_{\alpha=1}^{3} \ln F_{\alpha}^{e} F_{\alpha}^{p} \\
& =\sum_{\alpha=1}^{3} \ln F_{\alpha}^{e}+\sum_{\alpha=1}^{3} \ln F_{\alpha}^{p} \\
& =\operatorname{tr}\left(\ln \mathbf{V}^{e}\right)+\operatorname{tr}\left(\ln \mathbf{V}^{p}\right),
\end{aligned}
$$

where $F_{i}^{p}=\partial \bar{x}_{i} / \partial X_{i}, \quad F_{i}^{e}=\partial x_{i} / \partial \bar{x}_{i} \quad$ (no sum for $i$ ). That is to say, the additive decomposition holds in the volumetric strain for the Lee's multicative decomposition ${ }^{14)}$ of the deformation gradient.

The linear relation between both logarithms of the volume $v$ and the pressure $p$, i.e. the $\ln v-\ln p$ linear relation which would be physically pertinent and applicable to the description of the finite deformation was proposed by Hashiguchi ${ }^{8)-11)}$. From this relation the volumetric strain $\operatorname{tr}(\ln V)$, the elastic volumetric strain $\operatorname{tr}\left(\ln \mathbf{V}^{e}\right)$ and the plastic volumetric strain $\operatorname{tr}\left(\ln V^{p}\right)$ in the definition of logarithmic strain are given as follows (see Fig. 4):

$$
\begin{aligned}
& \operatorname{tr}(\ln \mathbf{V})=\operatorname{tr}\left(\ln \mathbf{V}^{e}\right)+\operatorname{tr}\left(\ln \mathbf{V}^{p}\right)=\ln \frac{v}{\bar{v}}+\ln \frac{\bar{v}}{V} \\
& =\ln \left(\frac{v}{\bar{v}}\right)+\left\{\ln \left(\frac{V_{y}}{V}\right)+\ln \left(\frac{v_{y}}{V_{y}}\right)+\ln \left(\frac{\bar{v}}{v_{y}}\right)\right\} \\
& =-\gamma \ln \left(\frac{p}{p_{0}}\right) \\
& \quad+\left\{-\gamma \ln \left(\frac{p_{y 0}}{p_{0}}\right)-\rho \ln \left(\frac{p_{y}}{p_{y 0}}\right)-\gamma \ln \left(\frac{p_{0}}{p_{y}}\right)\right\} \\
& =-\gamma \ln \left(\frac{p}{p_{0}}\right)-(\rho-\gamma) \ln \left(\frac{p_{y}}{p_{y 0}}\right),
\end{aligned}
$$

where

$$
p \equiv-\tau_{m},
$$

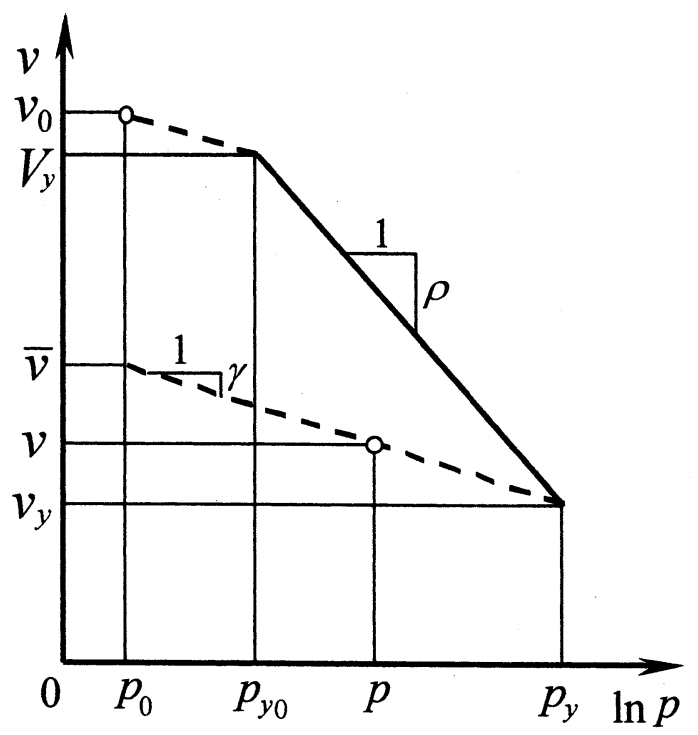

Fig. 4. The $\ln v-\ln p$ linear relation for isotropic consolidation of soils. 
$\rho$ and $\gamma$ are the slopes of normal-consolidation and swelling lines, respectively, in the $(\ln v, \ln p)$ plane. $p_{0}$ is the initial value of $p$, and $p_{y}$ and $p_{y 0}$ are the normal-consolidation pressure and its initial value, respectively.

Now, assume the following strain energy function

$$
\Psi=-\gamma p_{0} \exp \left(-\frac{\operatorname{tr}(\ln \mathbf{V})^{e}}{\gamma}\right)+G \operatorname{tr}\left((\ln \mathbf{V})^{e *}\right)^{2}
$$

where the elastic shear modulus $G$ is a function of the elastic volumetric strain $\operatorname{tr}(\ln \mathbf{V})^{e}$, i.e. $G\left(\operatorname{tr}(\ln \mathbf{V})^{e}\right)$. Eq. (51) is the generalization of the function proposed by Borja and Tamagnini ${ }^{30)}$ adopting the Lee's multicative decopmposition ${ }^{14)}$ of the deformation gradient and the limited form of the shear modulus. The stress is derived from Eq. (51) as

$$
\begin{aligned}
\boldsymbol{\tau} & =\left\{p_{0} \exp \left(-\frac{\operatorname{tr}(\ln \mathbf{V})^{e}}{\gamma}\right)\right. \\
& \left.+\frac{d G}{d \operatorname{tr}(\ln \mathbf{V})^{e}} \operatorname{tr}\left((\ln \mathbf{V})^{e *}\right)^{2}\right\} \mathbf{I}+2 G(\ln \mathbf{V})^{e *},
\end{aligned}
$$

where $\mathbf{I}$ is the second-order identity tensor. Eq. (52) coincides with Eq. (49) in the isotropic elastic deformation process. The stress rate is given from Eq. (52) as follows:

$$
\begin{aligned}
& \mathbf{t}=\left\{-\frac{p_{0}}{\gamma} \exp \left(-\frac{\operatorname{tr}(\ln \mathbf{V})^{e}}{\gamma}\right) \operatorname{tr}\left((\ln \mathbf{V})^{\circ}\right)^{e}\right. \\
& +\frac{d^{2} G}{d\left(\operatorname{tr}(\ln \mathbf{V})^{e}\right)^{2}}\left\{\operatorname{tr}\left((\ln \mathbf{V})^{e *}\right)^{2}\right\}\left\{\operatorname{tr}\left((\ln \mathbf{V})^{\circ}\right)^{e}\right\} \\
& +2 \frac{d G}{d \operatorname{tr}(\ln \mathbf{V})^{e}} \operatorname{tr}\left\{(\ln \mathbf{V})^{e *}\left((\ln \mathbf{V})^{\circ}\right)^{e}\right\} \\
& \left.-\frac{2}{3} G\left(\operatorname{tr}\left((\ln \mathbf{V})^{e}\right)^{\circ}\right)\right\} \mathbf{I} \\
& +2 \frac{d G}{d \operatorname{tr}(\ln \mathbf{V})^{e}}(\ln \mathbf{V})^{e *}\left\{\operatorname{tr}\left\{(\ln \mathbf{V})^{e}\right)^{\circ}\right\}+2 G\left((\ln \mathbf{V})^{\circ}\right)^{e}
\end{aligned}
$$

Then, the elastic modulus tensor is given as

$$
\begin{aligned}
\mathbf{E}= & \left\{-\frac{p_{0}}{\gamma} \exp \left(-\frac{\operatorname{tr}(\ln \mathbf{V})^{e}}{\gamma}\right)\right. \\
& \left.+\frac{d^{2} G}{d\left(\operatorname{tr}(\ln \mathbf{V})^{e}\right)^{2}}\left\{\operatorname{tr}\left((\ln \mathbf{V})^{e *}\right)^{2}\right\}-\frac{2 G}{3}\right\} \overline{\mathbf{I}} \\
& +2 \frac{d G}{d \operatorname{tr}(\ln \mathbf{V})^{e}} \mathbf{I} \otimes(\ln \mathbf{V})^{e *} \\
& +2 \frac{d G}{d \operatorname{tr}(\ln \mathbf{V})^{e}}(\ln \mathbf{V})^{e *} \otimes \mathbf{I}+2 G \tilde{\mathbf{I}}
\end{aligned}
$$

where

$$
\bar{I}_{i j k l} \equiv \delta_{i j} \delta_{k l}, \quad \tilde{I}_{i j k l} \equiv \delta_{i k} \delta_{j l}
$$

Transforming the Junbu's elastic shear modulus equation $^{12)}$ as the function of pressure

$$
G(p)=\zeta\left(\frac{p}{p_{0}}\right)^{n}
$$

to the function of the elastic volumetric strain due to Eq. (49), let the following function be assumed.

$$
G\left(\operatorname{tr}(\ln \mathbf{V})^{e}\right)=\zeta \exp \left(-\frac{n}{\gamma} \operatorname{tr}(\ln \mathbf{V})^{e}\right)
$$

where $\zeta$ and $n$ are material constants. It holds for Eq. (57) that

$$
\begin{aligned}
& \frac{d G}{d \operatorname{tr}(\ln \mathbf{V})^{e}}=-\zeta \frac{n}{\gamma} G, \\
& \frac{d^{2} G}{d\left(\operatorname{tr}(\ln \mathbf{V})^{e}\right)^{2}}=\left(\zeta \frac{n}{\gamma}\right)^{2} G
\end{aligned}
$$

\subsection{Material functions for inelastic constitutive equations}

In accordance with the previous articles ${ }^{4), 5)}$, 18),31),32) material function are described below.

Let the function $f$ in the subloading surface (15) be given for soils as

$$
f(\boldsymbol{\tau})=p\left\{1+\left(\frac{\|\boldsymbol{\eta}\|}{m}\right)^{2}\right\}
$$

where

$$
\boldsymbol{\eta} \equiv \frac{\boldsymbol{\tau}^{*}}{p}
$$

$m$ is a material function of Lode's angle or $b$-value and internal variables, representing the stress ratio in the critical state, whilst the simplest function for $m$ was given by Hashiguchi ${ }^{31)}$. || || stands for the magnitude.

The isotropic hardening/softening function $F$ is given from Eq. (49) as

$$
F=F_{0} \exp \left(\frac{H}{\rho-\gamma}\right),
$$

where $F_{0}$ is the initial value of $F$. Here, the evolution rule of isotropic hardening/softening variable $H$ is simply given by the plastic volumetric strain rate, i.e.

$$
\dot{H}=-\operatorname{tr}\left((\ln \mathbf{V})^{\circ}\right)^{p} .
$$

Let the function $\xi$ in equation (34) for the tangential-inelastic modulus $T$ be given as ${ }^{5}$

$$
\xi=\frac{p}{b \chi^{c}}
$$

where $b$ and $c$ are material constants.

\section{Concluding Remarks}

The unconventional elastoplastic constitutive 
equation with the tangential stress rate effect for large elastoplastic deformation of soils is formulated in this article, extending the large deformation theory of Naghdabadi and Saidi ${ }^{1)}$ and Hashiguchi et al. ${ }^{7)}$ as follows:

1 ) The corotational rate is adopted even for the elastic constitutive equation, while it has been ignored in the former formulation.

2 ) The Kirchhoff stress is adopted in stead of the Cauchy stress.

3 ) The hyper elastic constitutive equation is adopted in stead of the Cauchy elasticity.

Acknowledgements - The author expresses the sincere gratitude to Mr. A.R. Saidi, Sharif Univ. of Tech., Iran for kind advices and discussions, whilst he is staying in the author's laboratory from Dec. 16, 2002 to July 15, 2003.

\section{References}

1) Naghdabadi, R. and Saidi, A.R., Kinematic and constitutive modeling of elastic-plastic hardening materials based on logarithmic strain, Proc. 6th Biennial Conf. Eng. Systems Design and Anal, ASME-ESDA2002, Istanbul., pp. 1-8, 2002.

2) Hashiguchi, K., Constitutive equations of elastoplastic materials with elastic-plastic transition, $J$. Appl. Mech. (ASME), Vol.47, pp.266-272, 1980.

3) Hashiguchi, K., Subloading surface model in unconventional plasticity, Int. J. Solids Struct., Vol.25, pp. 917-945, 1989.

4) Hashiguchi, K., The tangential plasticity, Metals and Materials, Vol.4, pp. 652-656, 1998.

5) Hashiguchi, K. and Tsutsumi, S., Elastoplastic constitutive equation with tangential stress rate effect. Int. J. Plasticity, Vol.17, pp. 117-145, 2001.

6) Hashiguchi, K., Gradient elastoplastic constitutive equation extended to the subloading surface model with the tangential plasticity, Proc. 38th Annual Meeting of Japan Geotech. Soci., in press, 2003.

7) Hashiguchi, K., Saidi, A.R. and Naghdabadi, R., Large deformation theory by the logarithmic strain tensor and the subloading surface model with tangential plasticity, Proc. 58th Annual Meeting of Japan. Soci. Civil Engrs. , pp. 1127-1128, 2003.

8) Hashiguchi, K., Isotropic hardening theory of granular media, Proc. Japan Soc. Civil Eng., No. 227, pp. 45-60, 1974.

9) Hashiguchi, K. and Ueno, M., Elastoplastic constitutive laws of granular materials, Constitutive
Equations of Soils (Proc. 9th ICFSME, Spec. Session 9), Tokyo, JSSMFE, Tokyo, pp. 73-82, 1977.

10) Hashiguchi, K., On the linear relations of $V-\ln p$ and $\ln v-\ln p$ for isotropic consolidation of soils, Int. J. Numer. Anal. Meth. Geomech., Vol.19, pp. 367-376, 1995.

11) Hashiguchi, K. and Collins, I.F., Stress rate-elastic stretching relations in elastoplastic constitutive equations for soils, Soils and Foundations, Vol.41, No.2, pp. 77-87, 2001.

12) Junbu, N., Soil compressibility as determined by oedometer and triaxial test, European Conf. Soil Mech. Found. Eng., Wiesbaden, Vol.1, pp. 19-25, 1963.

13) Oldroyd, J.G., On the formulation of rheological equations of state, Proc. Royal Soci, London, Ser. $A$, Vol.200, 523-541, 1950.

14) Lee, E.H., Elastic-plastic deformation at finite strains, J. Appl. Mech. (ASME), Vol.36, pp.1-6, 1969.

15) Drucker, D.C., Conventional and unconventional plastic response and representation. Appl. Mech. Rev. (ASME), Vol.41, pp. 151-167, 1988.

16) Dafalias, Y.F., The plastic spin, J. Appl. Mech. (ASME), Vol.52, pp. 865-871, 1985.

17) Zbib, H.M., Aifantis, E.C., On the concept of relative and plastic spins and its implications to large deformation theories. Part I: Hypoelasticity and vertex-type plasticity, Acta Mech., Vol.75, pp.15-33, 1988.

18) Hashiguchi, K. and Chen, Z.-P., Elastoplastic constitutive equations of soils with the subloading surface and the rotational hardening, Int. J. Numer. Anal. Meth. Geomech., Vol.22, pp.197-227, 1998.

19) Hashiguchi, K., Description of inherent/induced anisotropy of soils: Rotational hardening rule with objectivity, Soils and Foundations, Vol.41, No.6, pp.139-145, 2001.

20) Hashiguchi, K., On the loading criterion, Int. J. Plasticity, Vol.10, pp.871-878, 1994.

21) Hashiguchi, K., Fundamentals in constitutive equation: continuity and smoothness conditions and loading criterion, Soils and Foundations, Vol.40, No.3, pp. 155-161, 2000.

22) Hashiguchi, K., Fundamental requirements and formulation of elastoplastic constitutive equations with tangential plasticity. Int. J. Plasticity, Vol.9, pp.525-549, 1993.

23) Hashiguchi, K., Mechanical requirements and structures of cyclic plasticity models. Int. J. Plasticity, Vol.9, pp.721-748, 1993. 
24) Hashiguchi, K., The extended flow rule in plasticity. Int. J. Plasticity, Vol.13, pp.37-58, 1997.

25) Rudnicki, J.W. and Rice, J.R., Conditions for localization of deformation in pressure-sensitive dilatant materials. J. Mech. Phys. Solids, Vol.23, pp.371-394, 1975.

26) Storen, S. and Rice, J.R., Localized necking in thin sheet. J. Mech. Phys. Solids, Vol.23, pp.421-441, 1975.

27) Vermeer, P., Upgrading of soil models by Hencky's theory of plasticity, Modern Approaches to Plasticity (ed. by D. Kolymbas), Elsevier, pp.71-82, 1993.

28) Papamichos, E., Vardoulakis, I. and Han, C., 1993. Noncoaxial flow theory of plasticity: shear failure prediction in sand, Modrern Approaches to Plasticity (ed. by D. Kolymbas), Elsevier pp.585-598, 1993.

29) Papamichos, E., Vardoulakis, I., Shear band formation in sand according to non-coaxial plasticity model. Geotechnique, Vol.45, pp.649-661, 1995.

30) Borja, R.I. and Tamagnini, C., Cam-clay plasticity Part III: Extension of the infinitesimal model to include finite strains. Compt. Meth. Appl. Eng., Vol.155, pp.73-95, 1998.

31) Hashiguchi, K., Saitoh, K., Okayasu, T. and Tsutsumi, S., Evaluation of typical conventional and unconventional plasticity models for prediction of softening behavior of soils, Geotechnique, Vol.52, pp.561-573, 2002.

32) Hashiguchi, K., A proposal of the simplest convex-conical surface for soils, Soils and Foundations, Vol.42, No.3, pp. 107-113, 2002.

\section{Appendix:}

Proof for the transformation of the material-time derivative to the corotational derivative of constitutive equation in rate form

Consider two different coordinate systems with bases $\left\{\mathbf{e}_{i}\right\}$ and $\left\{\mathbf{e}_{i}^{\prime}\right\}(i=1,2,3)$, whilst let the orthogonal tensor between these coordinate systems be denoted as $\mathbf{Q}$, i.e. $Q_{i j}=\mathbf{e}_{i}^{\prime} \bullet \mathbf{e}_{j}$, fulfilling $\mathbf{Q} \mathbf{Q}^{\mathrm{T}}=\mathbf{I}$ (see Fig. 1). The notation ( $)^{\mathrm{T}}$ stands for the transpose and $\mathbf{I}$ is the second-order identity tensor. The bases $\left\{\mathbf{e}_{i}\right\},\left\{\mathbf{e}_{i}^{\prime}\right\}$ and thus the orthogonal tensor $\mathbf{Q}$ depend on time in general. Let the position vector of material point observed by the coordinate systems with bases $\left\{\mathbf{e}_{i}\right\}$ and $\left\{\mathbf{e}_{i}^{\prime}\right\}$ be denoted $\mathbf{x}(\mathbf{X}, t)$ and
$\mathbf{x}^{\prime}(\mathbf{X}, t)$, respectively, where $\mathbf{X}$ is position vector of material point in the reference configuration and $t$ is a time. Then, it hold that

$$
\mathbf{x}^{\prime}(\mathbf{X}, t)=\mathbf{Q}(t) \mathbf{x}(\mathbf{X}, t)+\mathbf{c}(t),
$$

where $\mathbf{c}(t)$ is the position vector of the origin of the coordinate system with bases $\left\{\mathbf{e}_{i}\right\}$ observed in the coordinate system with bases $\left\{\mathbf{e}_{i}^{\prime}\right\}$.

The spin of material-substructure $\boldsymbol{\Omega}^{m}$ is generally related to the rotation tensor $\mathbf{R}^{m}$ of material-substructure as follows:

$$
\mathbf{\Omega}^{m}=\dot{\mathbf{R}}^{m} \mathbf{R}^{m \mathrm{~T}},
$$

provided that $\mathbf{R}^{m}$ is related to the motion $\mathbf{x}(\mathbf{X}, t)$ of material-substructure and fulfills the following transformation rule

$$
\mathbf{R}^{m^{\prime}}=\mathbf{Q R}^{m},
$$

and thus $\boldsymbol{\Omega}^{m}$ obeys the transformation rule

$$
\boldsymbol{\Omega}^{m \prime}=\mathbf{Q} \boldsymbol{\Omega}^{m} \mathbf{Q}^{\mathrm{T}}+\mathbf{\Omega},
$$

where $\boldsymbol{\Omega}$ is the spin of bases $\left\{\mathbf{e}_{i}\right\}$ observed from bases $\left\{\mathbf{e}_{i}^{\prime}\right\}$ as known from

$$
\mathbf{\Omega} \equiv\left(\dot{\mathbf{e}}_{r} \otimes \mathbf{e}_{r}\right)_{i j} \mathbf{e}_{i}^{\prime} \otimes \mathbf{e}_{j}^{\prime}=Q_{i r} Q_{j r} \mathbf{e}_{i}^{\prime} \otimes \mathbf{e}_{j}^{\prime}=\dot{\mathbf{Q}} \mathbf{Q}^{\mathrm{T}},
$$

noting $\dot{\mathbf{e}}_{i}=\mathbf{\Omega} \mathbf{e}_{i} \quad\left(\dot{\mathbf{e}}_{i} \otimes \mathbf{e}_{i}=\mathbf{\Omega} \mathbf{e}_{i} \otimes \mathbf{e}_{i}=\mathbf{\Omega}\right)$ and that the magnitudes of $\mathbf{e}_{i}$ are constant as unit.

Consider the constitutive equation relating the second-order tensors $\mathbf{T}$ and $\mathbf{S}$, which is described in the coordinated system with the bases $\left\{\mathbf{e}_{i}\right\}$ as follows.

$$
\mathbf{T}=\mathbf{f}(\mathbf{S}) \text {. }
$$

The time-differentiation of Eq. (A.1) leads to

$$
\dot{\mathbf{T}}=\dot{\mathbf{f}}(\mathbf{S})=\frac{\partial \mathbf{f}(\mathbf{S})}{\partial \mathbf{S}} \dot{\mathbf{S}}
$$

which has to be written in the coordinate system with the bases $\left\{\mathbf{e}_{i}^{\prime}\right\}$ as follows:

$$
\dot{\mathbf{T}}^{\prime}=\dot{\mathbf{f}}\left(\mathbf{S}^{\prime}\right)=\frac{\partial \mathbf{f}\left(\mathbf{S}^{\prime}\right)}{\partial \mathbf{S}^{\prime}} \dot{\mathbf{S}}^{\prime},
$$

with

$$
\stackrel{\bullet}{\mathbf{T}}^{\prime}=\mathbf{Q} \dot{\mathbf{T}} \mathbf{Q}^{\mathrm{T}}+\dot{\mathbf{Q}} \mathbf{T} \mathbf{Q}^{\mathrm{T}}+\mathbf{Q} \mathbf{T} \dot{\mathbf{Q}}^{\mathrm{T}}
$$

and

$$
\begin{aligned}
\frac{\partial \mathbf{f}\left(\mathbf{S}^{\prime}\right)}{\partial \mathbf{S}^{\prime}} \dot{\mathbf{S}}^{\prime}=\frac{\partial \mathbf{f}\left(\mathbf{Q S} \mathbf{Q}^{\mathrm{T}}\right)}{\partial \mathbf{Q S Q} \mathbf{Q}^{\mathrm{T}}} & \left(\mathbf{Q} \mathbf{S} \mathbf{Q}^{\mathrm{T}}+\dot{\mathbf{Q}} \mathbf{S} \mathbf{Q}^{\mathrm{T}}\right. \\
& \left.+\mathbf{Q S} \dot{\mathbf{Q}}^{\mathrm{T}}\right)
\end{aligned}
$$

from which one has

$$
\mathbf{Q}^{\mathrm{T}} \dot{\mathbf{T}}^{\prime} \mathbf{Q}=\mathbf{Q}^{\mathrm{T}}\left(\mathbf{Q} \dot{\mathbf{T}} \mathbf{Q}^{\mathrm{T}}+\dot{\mathbf{Q}} \mathbf{T} \mathbf{Q}^{\mathrm{T}}+\mathbf{Q} \mathbf{T} \dot{\mathbf{Q}}^{\mathrm{T}}\right) \mathbf{Q}
$$




$$
=\stackrel{\mathbf{T}}{+} \mathbf{Q}^{\mathrm{T}} \dot{\mathbf{Q}} \mathbf{T} \mathbf{Q}^{\mathrm{T}}+\mathbf{T} \dot{\mathbf{Q}}^{\mathrm{T}} \mathbf{Q} \quad(\mathrm{A} .11) \quad=\frac{\partial \mathbf{f}(\mathbf{S})}{\partial \mathbf{S}}\left(\dot{\mathbf{S}}+\mathbf{Q}^{\mathrm{T}} \dot{\mathbf{Q}} \mathbf{S}+\mathbf{S} \dot{\mathbf{Q}}^{\mathrm{T}} \mathbf{Q}\right) .
$$

and

$$
\begin{aligned}
& \mathbf{Q}^{\mathrm{T}} \frac{\partial \mathbf{f}\left(\mathbf{S}^{\prime}\right)}{\partial \mathbf{S}^{\prime}} \dot{\mathbf{S}}^{\prime} \mathbf{Q} \\
= & \mathbf{Q}^{\mathrm{T}} \frac{\partial \mathbf{f}\left(\mathbf{Q S} \mathbf{Q}^{\mathrm{T}}\right)}{\partial \mathbf{Q S} \mathbf{Q}^{\mathrm{T}}}\left(\mathbf{Q} \mathbf{S} \mathbf{Q}^{\mathrm{T}}+\dot{\mathbf{Q}} \mathbf{S} \mathbf{Q}^{\mathrm{T}}+\mathbf{Q} \mathbf{S} \dot{\mathbf{Q}}^{\mathrm{T}}\right) \mathbf{Q} \\
= & \mathbf{Q}^{\mathrm{T}} \frac{\partial \mathbf{f}\left(\mathbf{Q S S} \mathbf{Q}^{\mathrm{T}}\right)}{\partial \mathbf{Q S} \mathbf{Q}^{\mathrm{T}}} \mathbf{Q} \mathbf{Q}^{\mathrm{T}}\left(\mathbf{Q} \dot{\mathbf{S}} \mathbf{Q}^{\mathrm{T}}+\dot{\mathbf{Q}} \mathbf{S} \mathbf{Q}^{\mathrm{T}}\right. \\
& \left.+\mathbf{Q} \mathbf{S} \dot{\mathbf{Q}}^{\mathrm{T}}\right) \mathbf{Q}
\end{aligned}
$$

The selection of $\mathbf{Q}=\mathbf{R}^{m \mathrm{~T}}$ in Eqs. (A.11) and (A.12) leads to $\mathbf{Q}^{\mathrm{T}} \mathbf{Q}=\dot{\mathbf{R}}^{m} \mathbf{R}^{m \mathrm{~T}}=\mathbf{\Omega}^{m}$ and

$$
\stackrel{\circ}{\mathbf{T}}=\frac{\partial \mathbf{f}(\mathbf{S})}{\partial \mathbf{S}} \stackrel{\mathrm{S}}{\mathrm{S}}
$$

That is to say, the constitutive equation (A.7) in the form of material-time derivative can be transformed to that in the form of corotational derivative.

(Received April 18,2003) 